\title{
Simplicity of condensed matter at its core: Generic definition of a Roskilde-simple system
}

Schrøder, Thomas; Dyre, J. C.

Published in:

Journal of Chemical Physics

DOI:

10.1063/1.4901215

Publication date:

2014

\section{Document Version}

Publisher's PDF, also known as Version of record

Citation for published version (APA):

Schrøder, T., \& Dyre, J. C. (2014). Simplicity of condensed matter at its core: Generic definition of a Roskildesimple system. Journal of Chemical Physics, 141, [204502]. https://doi.org/10.1063/1.4901215

\section{General rights}

Copyright and moral rights for the publications made accessible in the public portal are retained by the authors and/or other copyright owners and it is a condition of accessing publications that users recognise and abide by the legal requirements associated with these rights.

- Users may download and print one copy of any publication from the public portal for the purpose of private study or research.

- You may not further distribute the material or use it for any profit-making activity or commercial gain.

- You may freely distribute the URL identifying the publication in the public portal.

\section{Take down policy}

If you believe that this document breaches copyright please contact rucforsk@kb.dk providing details, and we will remove access to the work immediately and investigate your claim. 


\title{
Simplicity of condensed matter at its core: Generic definition of a Roskilde-simple system
}

\author{
Thomas B. Schrøder ${ }^{\text {a) }}$ and Jeppe C. Dyre ${ }^{\text {b) }}$ \\ DNRF Centre "Glass and Time," IMFUFA, Department of Sciences, Roskilde University, Postbox 260, \\ DK-4000 Roskilde, Denmark
}

(Received 9 June 2014; accepted 24 October 2014; published online 25 November 2014)

\begin{abstract}
The isomorph theory is reformulated by defining Roskilde-simple systems by the property that the order of the potential energies of configurations at one density is maintained when these are scaled uniformly to a different density. If the potential energy as a function of all particle coordinates is denoted by $U(\mathbf{R})$, this requirement translates into $U\left(\mathbf{R}_{\mathrm{a}}\right)<U\left(\mathbf{R}_{\mathrm{b}}\right) \Rightarrow U\left(\lambda \mathbf{R}_{\mathrm{a}}\right)<U\left(\lambda \mathbf{R}_{\mathrm{b}}\right)$. Isomorphs remain curves in the thermodynamic phase diagram along which structure, dynamics, and excess entropy are invariant, implying that the phase diagram is effectively one-dimensional with respect to many reduced-unit properties. In contrast to the original formulation of the isomorph theory, however, the density-scaling exponent is not exclusively a function of density and the isochoric heat capacity is not an exact isomorph invariant. A prediction is given for the latter quantity's variation along the isomorphs. Molecular dynamics simulations of the Lennard-Jones and Lennard-Jones Gaussian systems validate the new approach. ( 2014 AIP Publishing LLC. [http://dx.doi.org/10.1063/1.4901215]
\end{abstract}

\section{INTRODUCTION}

In regard to structure and dynamics, liquids or solids dominated by van der Waals or weakly ionic and dipolar interactions, as well as metals, have much more regular behavior than condensed matter dominated by directional bonds (hydrogen or covalent bonds) or strong Coulomb forces. ${ }^{1-14}$ This old insight has recently been formalized and confirmed by computer simulations of several models systems. ${ }^{13}$ Thus it has been shown that systems with strong virial potentialenergy correlations-a characteristic of the former class of systems - have "isomorphic" curves in the condensed-matter region of the thermodynamic phase diagram, curves along which structure and dynamics in properly reduced units are invariant to a good approximation. The systems in question were first referred to as "strongly correlating," " but this name was often confused with strongly correlated quantum systems and now the term "Roskilde-simple systems" or just "Roskilde systems" is being used. ${ }^{12-21}$ The existence of isomorphs means that for many quantities the phase diagram becomes effectively one-dimensional, a property that rules out anomalies. ${ }^{11}$ A review of the isomorph theory was recently given in Ref. 13.

An important experimental signature of Roskilde-simple systems is that they obey power-law density scaling over limited density variations, i.e., that the relaxation time is a function of $\rho^{\gamma} / T$ where $\rho$ is the number density, $T$ is the temperature, and $\gamma$ is the so-called density-scaling exponent. ${ }^{22,23}$ These systems also obey isochronal superposition by which is meant the property that the average relaxation time determines the entire relaxation-time spectrum. ${ }^{24,25} \mathrm{~A}$ further application of the isomorph theory is the fact that for Roskilde-

\footnotetext{
a)Electronic mail: tbs@ ruc.dk

${ }^{b)}$ Electronic mail: dyre@ ruc.dk
}

simple systems the melting line is an isomorph, which explains the invariances along it of several quantities. ${ }^{26-29}$

A system of $N$ particles in volume $V$ is considered with $\rho \equiv N / V$. The theory of isomorphs refers to quantities given in so-called reduced units. ${ }^{27}$ The length and energy units are always $\rho^{-1 / 3}$ and $k_{B} T$, respectively, whereas the time unit depends on the dynamics (Newtonian or Brownian). In terms of the particle coordinates the configuration vector is defined by $\mathbf{R} \equiv\left(\mathbf{r}_{1}, \ldots, \mathbf{r}_{N}\right)$; its reduced-unit version is given by $\tilde{\mathbf{R}} \equiv \rho^{1 / 3} \mathbf{R}$. The original isomorph theory ${ }^{27}$ defines two thermodynamic state points with density and temperature $\left(\rho_{1}\right.$, $\left.T_{1}\right)$ and $\left(\rho_{2}, T_{2}\right)$, respectively, to be isomorphic if the following condition is obeyed: Whenever two physically important configurations of the state points, $\mathbf{R}_{1}$ and $\mathbf{R}_{2}$, have the same reduced coordinates, i.e., $\rho_{1}^{1 / 3} \mathbf{R}_{1}=\rho_{2}^{1 / 3} \mathbf{R}_{2}$, the following applies:

$$
\exp \left(-U\left(\mathbf{R}_{1}\right) / k_{B} T_{1}\right) \cong C_{12} \exp \left(-U\left(\mathbf{R}_{2}\right) / k_{B} T_{2}\right) .
$$

It is understood that the constant $C_{12}$ does not depend on the configurations. Thus whenever two configurations of isomorphic state points have the same reduced coordinates, their canonical probabilities are (almost) identical. This implies (almost) identical structure and dynamics in reduced units. ${ }^{13,27}$

It was recently shown that the existence of isomorphs for a given system is conveniently expressed in the "hidden-scaleinvariance" identity that factorizes the potential-energy function $U(\mathbf{R})$ as follows: ${ }^{13,30}$

$$
U(\mathbf{R}) \cong h(\rho) \tilde{\Phi}(\tilde{\mathbf{R}})+g(\rho) .
$$

Here the function $\tilde{\Phi}(\tilde{\mathbf{R}})$ is dimensionless and state-point independent. Equation (2) expresses a global, approximate scale invariance in the sense that the function $\tilde{\Phi}(\tilde{\mathbf{R}})$, which determines structure and dynamics in reduced coordinates, is unchanged for a uniform scaling of all particle coordinates. 
This global approximate scale invariance is in the present paper modified into a more local form of scale invariance valid along each isomorph separately.

The theory proposed below does not drastically change the predictions of the original isomorph theory,${ }^{27}$ but there are some important differences. In Sec. II, we present an intuitive approach emphasizing the underlying physical idea. Section III proceeds axiomatically and derives the generalized isomorph theory from a new definition of Roskilde-simple systems. Because of its axiomatic approach Sec. III can be read independently of Sec. II. Section IV establishes the connection between the two approaches and finally Sec. V summarizes the paper.

\section{TOWARDS A GENERALIZED ISOMORPH THEORY: AN INTUITIVE APPROACH}

For numerical tests it is convenient to transform Eq. (2) into an equation relating the potential-energy surfaces at two different densities, $\rho_{1}$ and $\rho_{2}$. In the following, we let $\mathbf{R}_{1}$ and $\mathbf{R}_{2}$ denote configurations at densities $\rho_{1}$ and $\rho_{2}$, respectively, which have identical reduced coordinates, i.e., $\rho_{1}^{1 / 3} \mathbf{R}_{1}=\rho_{2}^{1 / 3} \mathbf{R}_{2} \equiv \tilde{\mathbf{R}}$. By elimination of $\tilde{\Phi}(\tilde{\mathbf{R}})$ Eq. (2) implies

$$
U\left(\mathbf{R}_{2}\right) \cong h_{1}\left(\rho_{2}\right) U\left(\mathbf{R}_{1}\right)+g_{1}\left(\rho_{2}\right) .
$$

Here the functions $h_{1}\left(\rho_{2}\right)$ and $g_{1}\left(\rho_{2}\right)$ depend on $\rho_{1}$, which henceforth plays the role of reference density for which reason the $\rho_{1}$ dependence is only indicated in the subscript 1 . Equation (3) describes how the potential-energy surface at density $\rho_{1}$ scales when density is changed to $\rho_{2}$, namely, to

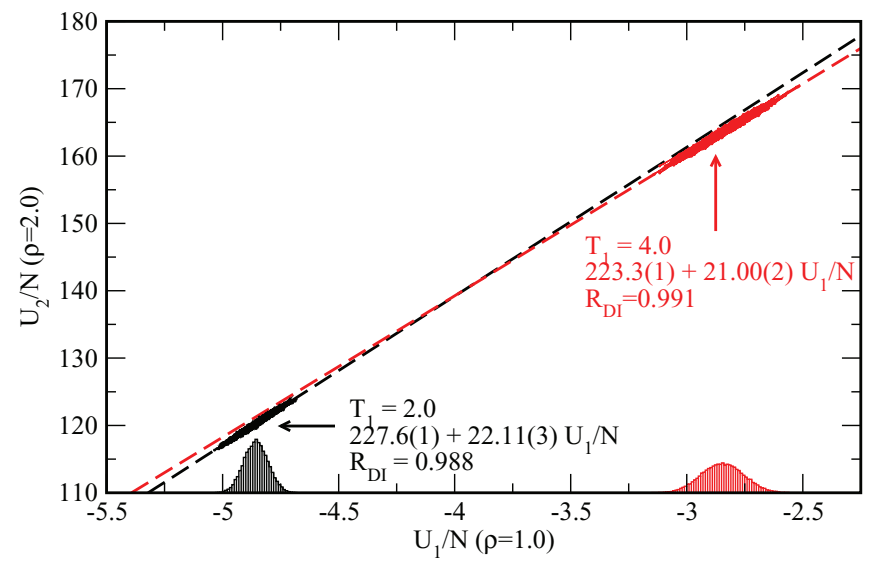

FIG. 1. Results from uniform scaling of configurations of a Lennard-Jones (LJ) liquid from density 1.0 to density 2.0 (in the LJ unit system defined by $\epsilon=\sigma=1$ ). Black gives a scatter plot for configurations generated at temperature 2.0 and red at temperature 4.0 , in both cases from simulations at the reference density $\rho_{1}=1.0$. Dashed lines are linear regression fits, numbers in parentheses indicate the estimated error on the last digit. $\mathrm{R}_{\mathrm{DI}}$ is the Pearson correlation coefficient for the two data sets. The strong correlations between original, $U_{1} \equiv U\left(\mathbf{R}_{1}\right)$, and scaled potential energies, $U_{2} \equiv U\left(\mathbf{R}_{2}\right)$, confirms that the LJ liquid is a Roskilde-simple system, i.e., one with strong virial potential-energy correlations and good isomorphs. ${ }^{27}$ The distributions of $U_{1}$ for the temperatures 2.0 and 4.0, respectively, are indicated on the xaxis. $1728 \mathrm{LJ}$ particles were simulated in the $N V T$ ensemble using a NoseHoover thermostat with time constant 0.2. The time step was 0.001 and the potential was cut and shifted at 4.5 (LJ units). The simulations were carried out using the Roskilde University Molecular Dynamics (RUMD) code. ${ }^{32}$
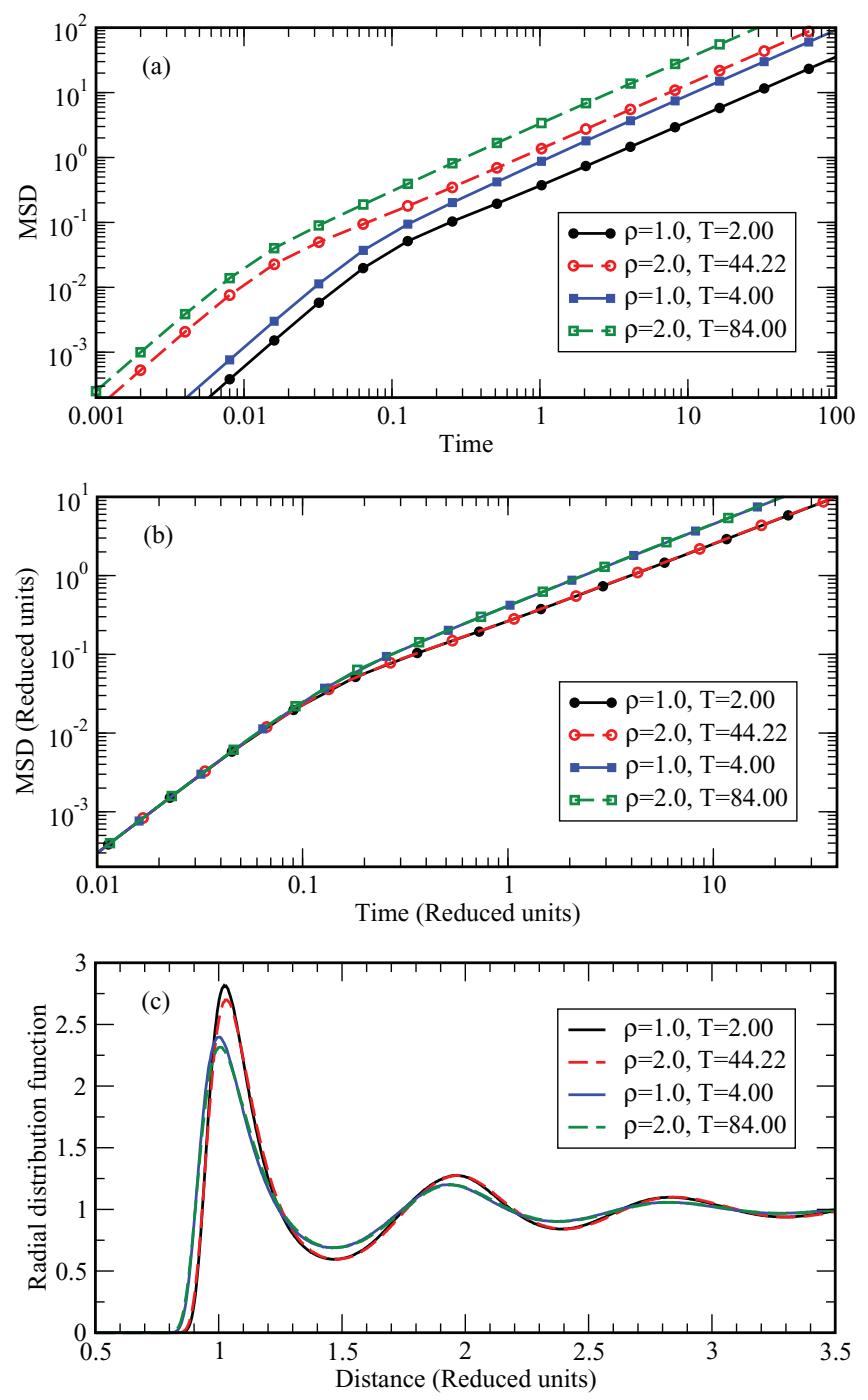

FIG. 2. Investigations of the isomorph invariance of structure and dynamics of the LJ system for the state points of Fig. 1 according to which $(\rho, T)=$ $(1.0,2.0)$ is predicted to be isomorphic to the state point $(2.0,2.0 \cdot 22.11)$ $=(2.0,44.22)$ since the linear regression slope of the direct isomorph check (black points) in Fig. 1 is 22.11. Similarly, $(\rho, T)=(1.0,4.0)$ is predicted to be isomorphic to $(2.0,84.00)$. (a) Mean-square displacement in standard LJ units for the four state points. (b) Mean-square displacement in reduced units, demonstrating isomorph invariance. ${ }^{27}$ (c) Radial distribution functions in reduced units, demonstrating isomorph invariance with minor deviations at the first peak maximum.

a good approximation simply by a linear, affine transformation. In particular, Eqs. (3) implies (1) if the temperatures involved obey $T_{2} / T_{1}=h_{1}\left(\rho_{2}\right)=h\left(\rho_{2}\right) / h\left(\rho_{1}\right),{ }^{13,27}$ which is thus the condition for identifying isomorphic state points. This observation forms the basis of the so-called "direct isomorph check" 27 in which configurations drawn from an equilibrium simulation at $\rho_{1}$ are used to test the scaling by evaluating the potential energy after uniformly scaling the configurations to density $\rho_{2}$. As an example, in Fig. 1 this is done for the singlecomponent Lennard-Jones (LJ) system with $\rho_{1}=1.0$ and $\rho_{2}$ $=2.0$ (LJ units). The black and red points refer to drawing $\mathbf{R}_{1}$ 's from equilibrium simulations at $T_{1}=2.0$ and $T_{1}=4.0$, respectively (LJ units). The original isomorph theory implying Eq. (3) predicts these two scatter plots to lie on a common straight line. This applies approximately, but not exactly. Thus 

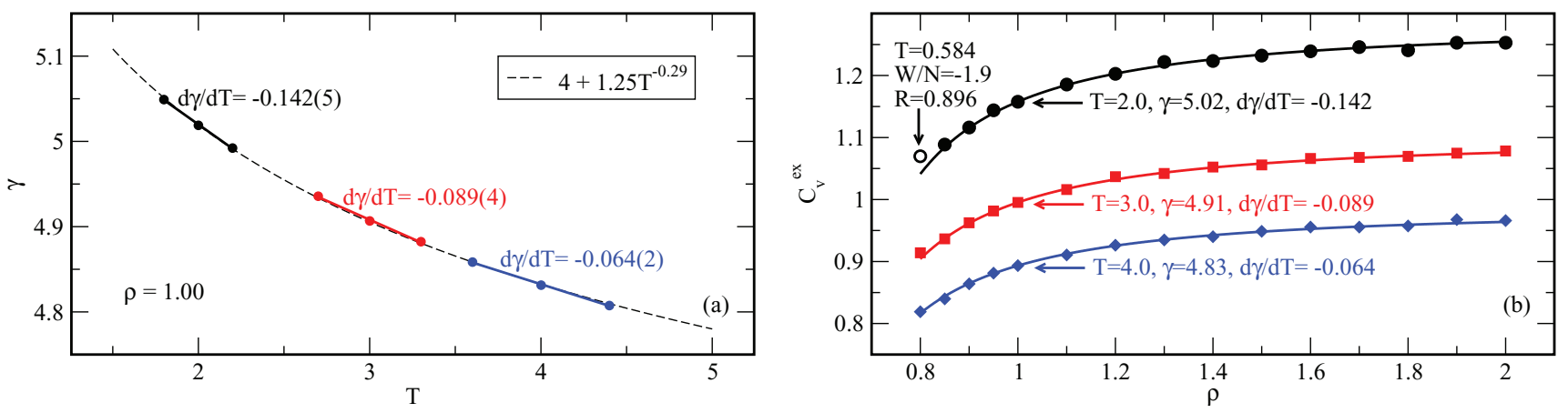

FIG. 3. (a) Density-scaling exponent $\gamma$ of the LJ system calculated from the fluctuation expression ${ }^{27} \gamma=\langle\Delta W \Delta U\rangle /\left\langle(\Delta U)^{2}\right\rangle$ at density 1.0 as a function of temperature, a quantity the original isomorph theory predicts to only depend on the density. ${ }^{27}$ Dashed curve: a power law plus 4 (the known high-temperature limit coming from the LJ potential's $r^{-12}$ term) plotted as a guide to the eye. (b) Excess isochoric heat capacity along three different isomorphs, a quantity the original isomorph theory predicts to be isomorph invariant. ${ }^{27}$ The full curves are the predictions of the new formulation of the isomorph theory (Eq. (6) with $h_{1}\left(\rho_{2}\right)=\left(\rho_{2} / \rho_{1}\right)^{4}\left(\gamma_{1} / 2-1\right)-\left(\rho_{2} / \rho_{1}\right)^{2}\left(\gamma_{1} / 2-2\right)$ with $\gamma_{1} \equiv \gamma\left(\rho_{1}, T_{1}\right)$, a function that can be determined from the simulation data obtained at the reference density $\rho_{1}=1.0$ reported in (a) (this functional form is specific for the LJ system ${ }^{33,34}$ ). As low densities are approached, the theory breaks down. For the lowest temperature we included one data point where this starts to happen (open black circle). This point is a metastable liquid in the gas-liquid co-existence region; it has negative virial and a lower virial potential-energy correlation coefficient $R$-these three properties all indicate breakdown of the isomorph theory. ${ }^{27}$

doubling the sampling temperature from 2.0 to 4.0 , changes the estimated value of $h_{1}\left(\rho_{2}\right)$ by roughly $5 \%$. As demonstrated below, such small deviations have significant consequences for the variation of the isochoric heat capacity $C_{V}$ along the isomorphs ${ }^{31}$ and for the temperature dependence of the density-scaling exponent at fixed density.

In order to generalize Eq. (3), we assume a general oneto-one mapping of the potential-energy surface at $\rho_{1}$ to that at $\rho_{2}$,

$$
U\left(\mathbf{R}_{2}\right) \cong f_{1}\left(\rho_{2}, U\left(\mathbf{R}_{1}\right)\right) .
$$

The original formulation of the isomorph theory as expressed in Eq. (3) is recovered as the first-order Taylor approximation to Eq. (4). Consider a direct isomorph check corresponding, e.g., to the black points in Fig. $1\left(T_{1}=2.0\right)$. For the relevant range of potential energies Eq. (3) is evidently an excellent approximation to Eq. (4) if one identifies

$$
h_{1}\left(\rho_{2}, U_{1}\right) \equiv\left(\frac{\partial f_{1}\left(\rho_{2}, U_{1}\right)}{\partial U_{1}}\right)_{\rho_{1}, \rho_{2}}
$$

in which $U_{1}$ is the mean potential energy at the reference state point $\left(\rho_{1}, T_{1}\right)$. Defining $T_{2} \equiv h_{1}\left(\rho_{2}, U_{1}\right) T_{1}$, the state point $\left(\rho_{2}, T_{2}\right)$ is isomorphic to the state point $\left(\rho_{1}, T_{1}\right)$, compare the discussion above after Eq. (3). Following Ref. 27, it is straightforward to show that:

1. The canonical probabilities of the configurations $\mathbf{R}_{1}$ and $\mathbf{R}_{2}$ are identical (to a good approximation), implying that all structural characteristics are invariant in reduced units; ${ }^{27}$

2. the reduced forces associated with the configurations $\mathbf{R}_{1}$ and $\mathbf{R}_{2}$ are identical, which implies that the dynamics is isomorph invariant in reduced units. The predicted isomorph invariance of structure and dynamics for the state points of Fig. 1 is confirmed in Fig. 2;

3. since the excess entropy is determined by the structure, this quantity is an isomorph invariant.

An obvious question is: Are there corrections to these three points coming from the fact that they were derived from a first-order approximation to Eq. (4)? Based on considerations of the dependence on the system size $N$, this cannot be the case: The range of potential energies sampled at $\left(\rho_{1}, T_{1}\right)$ depends on the system size. The standard deviation of $U_{1} / N$ is proportional to $1 / \sqrt{N}$, i.e., had we simulated a four times larger system, the distributions in Fig. 1 would have been half as wide. Thus approaching the thermodynamic limit, the firstorder approximation to Eq. (4) becomes better and better; in other words, the three above predictions are not influenced by the higher-order derivatives of Eq. (4) since the predictions deal (implicitly) with the thermodynamic limit.

But which predictions do change in the new formulation of the isomorph theory, Eq. (4)? In the formulation Eq. (3), the ratio $T_{2} / T_{1}$ is given by $h_{1}\left(\rho_{2}\right)$, which only depends on the densities involved, $\rho_{1}$ and $\rho_{2}$. In the new formulation, this ratio is given by $h_{1}\left(\rho_{2}, U_{1}\right)$ and in general depends on the isomorph in question-parameterized by $U_{1}$, the potential energy at the reference density $\rho_{1}$. It follows that the density-scaling exponent $^{27} \gamma \equiv(\partial \ln T / \partial \ln \rho)_{S_{\text {ex }}}$ may vary on the isochores, whereas in the original isomorph theory $\gamma$ was predicted to be constant on these. ${ }^{27}$ Figure 3(a) shows that $\gamma$ indeed does change on the $\rho=1$ isochore, slowly approaching the limit 4 known to apply at very high temperatures at which the LJ potential's repulsive $r^{-12}$ term dominates.

Many thermodynamic response functions get a contribution from the second derivatives of $f_{1}\left(\rho_{2}, U_{1}\right)$ - the exceptions being those for which the excess entropy is kept constant. An important case is the excess isochoric heat capacity, $C_{V}^{\mathrm{ex}}$, which is predicted to be isomorph invariant in the original formulation of the theory ${ }^{27}$ though this is not always accurately obeyed in simulations. ${ }^{31}$ Writing $C_{V, 2}^{\mathrm{ex}}=\left(\partial U_{2} / \partial T_{2}\right)_{\rho_{2}}$ $=\left(\partial U_{2} / \partial U_{1}\right)_{\rho_{2}}\left(\partial U_{1} / \partial T_{2}\right)_{\rho_{2}}$ and using $T_{2}=h_{1}\left(\rho_{2}, U_{1}\right) T_{1}$ in conjunction with Eq. (5), it is straightforward to show that $1 / C_{V, 2}^{\mathrm{ex}}=1 / C_{V, 1}^{\mathrm{ex}}+\left(T_{1} / h_{1}\left(\rho_{2}, U_{1}\right)\right)\left(\partial h_{1}\left(\rho_{2}, U_{1}\right) / \partial U_{1}\right)_{\rho_{2}}$. This can be rewritten as

$$
C_{V, 2}^{\mathrm{ex}}=C_{V, 1}^{\mathrm{ex}} /\left[1+\left(\frac{\partial \ln h_{1}\left(\rho_{2}, U_{1}\right)}{\partial \ln T_{1}}\right)_{\rho_{1}, \rho_{2}}\right] .
$$



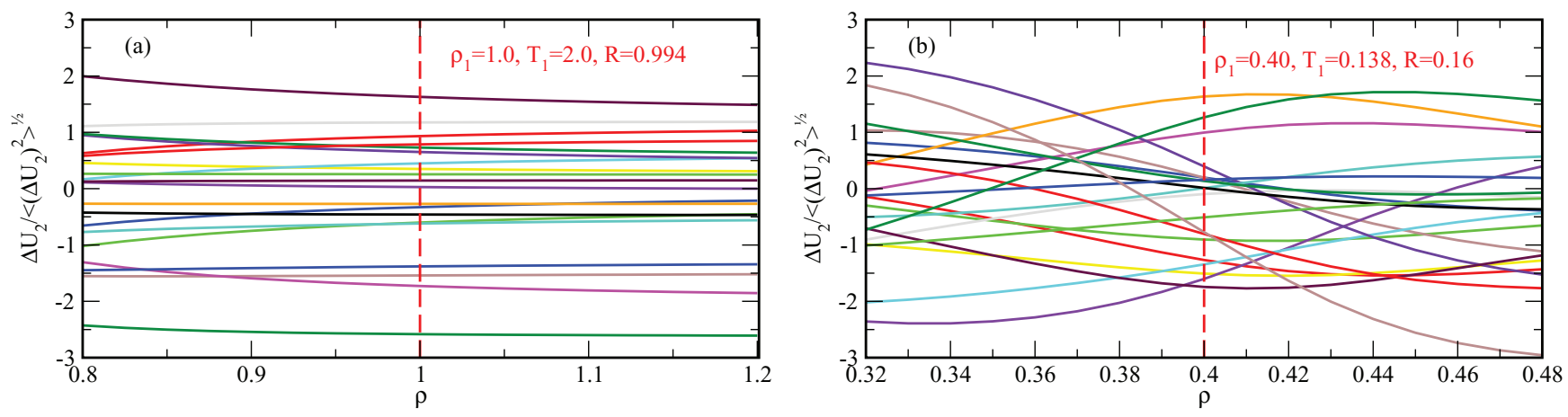

FIG. 4. Illustrations of the new definition of a Roskilde-simple system. Each figure shows the potential energies of 20 configurations taken from an equilibrium simulation at the density marked by the red dashed line, which were subsequently scaled $20 \%$ uniformly up and down in density and plotted as a function of density after being normalized by subtracting the average potential energy and scaled by the standard deviation (averages and standard deviations were determined from 1000 configurations). For an ideal Roskilde-simple system, the curves cannot cross each other. (a) Data for the LJ system based on simulations at the state point $(\rho, T)=(1.0,2.0)$ where this system has strong virial potential-energy correlations $(R=0.99)$. (b) Data for the Lennard-Jones Gaussian (LJG) system $^{35}$ based on simulations at the state point $(\rho, T)=(0.4,0.138)$ where $R=0.16$.

For LJ systems an analytical expression for $h_{1}\left(\rho_{2}, U_{1}\right)$ has been derived: $h_{1}\left(\rho_{2}\right)=\left(\rho_{2} / \rho_{1}\right)^{4}\left(\gamma_{1} / 2-1\right)-\left(\rho_{2} / \rho_{1}\right)^{2}\left(\gamma_{1} / 2\right.$ - 2) with $\gamma_{1} \equiv \gamma\left(\rho_{1}, T_{1}\right),{ }^{33,34}$ which combined with Eq. (6) shows that the variation of $C_{V}^{\mathrm{ex}}\left(\rho_{2}\right)$ along an isomorph is determined by the two numbers $\gamma_{1}$ and $\left(\partial \gamma_{1} / \partial T\right)_{\rho_{1}}$. Figure 3(b) tests the prediction for $C_{V, 2}^{\mathrm{ex}}$ for three isomorphs generated with $T_{1}=2.0,3.0$, and 4.0, respectively, at the reference density $\rho_{1}=1.0$. The values of $\gamma_{1}$ and $\left(\partial \gamma_{1} / \partial T\right)_{\rho_{1}}$ were determined from the reference density simulations reported in Fig. 3(a). As can be seen in Fig. 3(b) the $C_{V}^{\mathrm{ex}}\left(\rho_{2}\right)$ prediction agrees very well with the simulations.

\section{AXIOMATIC FORMULATION}

In Sec. II an isomorph was identified by the potential energy at the reference density $\rho_{1}$, which is expedient in numerical tests of the theory. In this section, we formalize the new theory, and here it is more convenient to identify the isomorphs by their excess entropy.

A Roskilde-simple system is henceforth defined by the property that whenever two configurations $\mathbf{R}_{a}$ and $\mathbf{R}_{b}$ refer to the same density, one has

$$
U\left(\mathbf{R}_{\mathrm{a}}\right)<U\left(\mathbf{R}_{\mathrm{b}}\right) \Rightarrow U\left(\lambda \mathbf{R}_{\mathrm{a}}\right)<U\left(\lambda \mathbf{R}_{\mathrm{b}}\right)
$$

Because this will apply for scaling "both ways," an equivalent formulation is to replace $\Rightarrow$ by $\Leftrightarrow$, which in turn implies that if two configurations have the same potential energy, their scaled versions also have same potential energy. Thus an equivalent definition of a Roskilde-simple systems is

$$
U\left(\mathbf{R}_{\mathrm{a}}\right)=U\left(\mathbf{R}_{\mathrm{b}}\right) \Rightarrow U\left(\lambda \mathbf{R}_{\mathrm{a}}\right)=U\left(\lambda \mathbf{R}_{\mathrm{b}}\right)
$$

Any Euler-homogeneous potential-energy function obeys this condition, but so does a homogeneous function plus a constant; in view of this Eq. (8) may be said to express a generalized homogeneity condition. Presumably no other systems obey Eq. (8) for all configurations. In the following, we make the weaker assumption that Eqs. (7) or (8) apply for most of the physically relevant configurations. This reflects the fact that the isomorph theory is inherently approximate for all realistic models.

Figure 4 shows the potential energies as functions of density for scaled configurations of the LJ system that has strong virial potential-energy correlations in the dense fluid phase, as well as for the Lennard-Jones Gaussian (LJG) system for which this is not the case. For both systems 20 configurations were picked from an equilibrium simulation at the density marked by the red vertical dashed line, and each of these configurations were scaled uniformly to densities involving changes of $\pm 20 \%$. According to Eq. (7) curves giving the potential energy of such uniformly scaled configurations cannot cross each other. Since compression increases the potential energy dramatically, in order to facilitate comparison with Eq. (7) at each density we subtracted the mean potential energy and scaled by the standard deviation-still, a system is (perfectly) Roskilde-simple if no curves cross each other. This is obeyed to a good approximation for the LJ system, but not for the LJG system; the low-density weak violations observed for the LJ system reflect the fact that it here gradually enters a region of weaker virial potential-energy correlations (compare Fig. 3(b)).

In the remainder of Sec. III, whenever a thermodynamic quantity is given without reference to a specific configuration $\mathbf{R}$, the quantity refers to the thermal equilibrium value at the state point in question. When we write $U$, for instance, this means the average potential energy at the given state point, whereas writing $U(\mathbf{R})$ signifies the potential energy of the configuration $\mathbf{R}$.

Recall that the entropy $S$ may be written as a sum of the ideal-gas entropy $S_{\text {id }}$ at the same density and temperature and the so-called excess entropy, $S=S_{\text {id }}+S_{\text {ex }}$. For an ideal gas $S_{\text {ex }}=0$; for any system with interactions $S_{\text {ex }}<0$ because no matter what is the nature of the interactions, such a system must be less disordered than an ideal gas. The Appendix reviews the definition of the excess free energy and other excess thermodynamic quantities; it also reviews the derivation of the microcanonical ensemble expression for $S_{\mathrm{ex}}$, which is used below for developing the new formulation of the isomorph theory. 
In their theory of thermodynamic fluctuations, Landau and Lifshitz define the entropy fluctuation at a given state point as the change in the equilibrium entropy "formally regarded as a function of the exact value of the fluctuating energy." 36 More generally, given a system and a set of coarsegrained variables $A_{1}, \ldots, A_{n}$, for any given microstate $\Gamma$ one defines (via an integral over delta functions) the entropy function $S(\Gamma)$ as the logarithm of the number of microstates that have the same coarse-grained variables as $\Gamma .{ }^{37}$ Clearly, $S(\Gamma)$ depends on the choice of coarse-grained variables. The case discussed by Landau and Lifshitz corresponds to that of a single coarse-grained variable, the energy; we follow this below except for using the potential energy instead of the total energy as coarse-grained variable.

We thus define the microscopic excess entropy function $S_{\mathrm{ex}}(\mathbf{R})$ as the thermodynamic excess entropy of a system with average potential energy equal to $U(\mathbf{R})$ at the density $\rho$ of the configuration $\mathbf{R}$, i.e.,

$$
S_{\mathrm{ex}}(\mathbf{R}) \equiv S_{\mathrm{ex}}(\rho, U(\mathbf{R})) .
$$

Here $S_{\mathrm{ex}}(\rho, U)$ is the thermodynamic equilibrium excess entropy of the state point with density $\rho$ and average potential energy $U$. This definition only makes sense for typical configurations filling out the volume, of course, because it is essential that the configuration defines a density. Inverting Eq. (9), the potential-energy function by definition obeys

$$
U(\mathbf{R})=U\left(\rho, S_{\mathrm{ex}}(\mathbf{R})\right)
$$

in which the right-hand side is the thermodynamic equilibrium potential energy as a function of density $\rho$ and thermodynamic excess entropy $S_{\mathrm{ex}}$, evaluated by substituting $S_{\text {ex }}=S_{\text {ex }}(\mathbf{R})$.

By definition, Eqs. (9) and (10) apply for any system. We now limit the discussion to Roskilde-simple systems. Suppose $\mathbf{R}_{1}$ is a configuration at density $\rho_{1}$ with the same reduced coordinate as $\mathbf{R}_{2}$, a configuration at density $\rho_{2}$. It follows from Eq. (9) and the microcanonical expression for the excess entropy (Eq. (A1) of the Appendix) that if "Vol" is the reducedcoordinate configuration-space volume, one has

$$
\begin{aligned}
& S_{\text {ex }}\left(\mathbf{R}_{1}\right) / k_{B} \\
& \quad=-N \ln N+\ln \left(\operatorname{Vol}\left\{\tilde{\mathbf{R}}^{\prime} \mid U\left(\rho_{1}^{-1 / 3} \tilde{\mathbf{R}}^{\prime}\right)<U\left(\mathbf{R}_{1}\right)\right\}\right) .
\end{aligned}
$$

Likewise

$$
\begin{aligned}
& S_{\mathrm{ex}}\left(\mathbf{R}_{2}\right) / k_{B} \\
& \quad=-N \ln N+\ln \left(\operatorname{Vol}\left\{\tilde{\mathbf{R}}^{\prime} \mid U\left(\rho_{2}^{-1 / 3} \tilde{\mathbf{R}}^{\prime}\right)<U\left(\mathbf{R}_{2}\right)\right\}\right) .
\end{aligned}
$$

Because $\mathbf{R}_{2}=\rho_{2}^{-1 / 3} \rho_{1}^{1 / 3} \mathbf{R}_{1}$, applying $\lambda=\rho_{2}^{-1 / 3} \rho_{1}^{1 / 3}$ in the $\Leftrightarrow$ version of Eq. (7) to the inequality of the first set (Eq. (11)) we see that the two sets are identical. Thus $S_{\text {ex }}\left(\mathbf{R}_{1}\right)=S_{\text {ex }}\left(\mathbf{R}_{2}\right)$, which means that for a Roskilde-simple system $S_{\mathrm{ex}}(\mathbf{R})$ depends only on the configuration's reduced coordinate

$$
S_{\text {ex }}(\mathbf{R})=S_{\text {ex }}(\tilde{\mathbf{R}}) .
$$

Equation (10) thus becomes

$$
U(\mathbf{R})=U\left(\rho, S_{\mathrm{ex}}(\tilde{\mathbf{R}})\right) .
$$

This " $U=U$ " relation, which links the microscopic potentialenergy function to the thermodynamic average potentialenergy function, gives the fundamental characterization of Roskilde-simple systems. It is understood that, just as in the original isomorph theory, this identity is not obeyed exactly for all configurations, but to a good approximation for most of the physically relevant configurations. We proceed to derive the consequences of Eq. (14).

\section{A. Invariance of structure and dynamics along the configurational adiabats}

In reduced coordinates Newton's second law for a system of identical masses is $d^{2} \tilde{\mathbf{R}} / d \tilde{t}^{2}=\tilde{\mathbf{F}}$ in which the reduced force vector is defined from the full force vector $\mathbf{F}$ (all particle forces joined into a single vector) by $\tilde{\mathbf{F}} \equiv \mathbf{F} \rho^{-1 / 3} / k_{B} T^{27}$ (the below derivations all generalize straight away to systems of particles with different masses).

In general, one has $\tilde{\mathbf{F}}=\tilde{\mathbf{F}}(\mathbf{R})$, implying different dynamics at different state points. For a Roskilde-simple system, however, as we shall see now, the reduced force is a function of the reduced configuration vector, $\tilde{\mathbf{F}}=\tilde{\mathbf{F}}(\tilde{\mathbf{R}})$. To show this, note that since $\nabla=\rho^{1 / 3} \tilde{\nabla}$, Eq. (14) implies $\mathbf{F}=-\nabla U$ $=-\left(\partial U / \partial S_{\text {ex }}\right)_{\rho} \rho^{1 / 3} \tilde{\nabla} S_{\text {ex }}(\tilde{\mathbf{R}})$. Since $\left(\partial U / \partial S_{\text {ex }}\right)_{\rho}=T$, this means that $\tilde{\mathbf{F}}=-\tilde{\nabla} S_{\mathrm{ex}}(\tilde{\mathbf{R}}) / k_{B}$. Thus the reduced force is a unique function of the reduced coordinates. This implies that the reduced-unit dynamics is invariant along the configurational adiabats, because via Eq. (13) two state points on a given isomorph—given by a certain value of $S_{\mathrm{ex}}$-correspond to the same reduced coordinate vectors $\tilde{\mathbf{R}}$.

The fact that the dynamics is invariant along the configurational adiabats immediately implies invariance of the reduced-unit structure: If the same configurations are traced out in the course of time at two different state points-except for a uniform scaling of space and time- the structure as measured, e.g., via the reduced-unit radial distribution function or higher-order correlation functions must be identical at the two state points. This is of course consistent with the fact that $S_{\mathrm{ex}}$, a measure of the structural disorder, by definition is constant along the configurational adiabats.

\section{B. Isomorphs}

Inspired by the above we now define isomorphs as the configurational adiabats in the thermodynamic phase diagram. Thus by definition the excess entropy is an isomorph invariant. As we have seen in Sec. III A, structure and dynamics are invariant to a good approximation along the isomorphs.

Expanding Eq. (14) to first order at constant density at any given state point and recalling that $\left(\partial U / \partial S_{\text {ex }}\right)_{\rho}=T$ we get

$$
U(\mathbf{R}) \cong U+T\left(\rho, S_{\mathrm{ex}}\right)\left(S_{\mathrm{ex}}(\tilde{\mathbf{R}})-S_{\mathrm{ex}}\right) .
$$

Consider two state points $\left(\rho_{1}, T_{1}\right)$ and $\left(\rho_{2}, T_{2}\right)$ with the same excess entropy $S_{\text {ex }}$. If $\mathbf{R}_{1}$ and $\mathbf{R}_{2}$ are two physically relevant configurations of these state points with the same reduced coordinates, Eq. (15) implies that if one for brevity writes 
$T\left(\rho_{1}, S_{\mathrm{ex}}\right)=T_{1}$ and $T\left(\rho_{2}, S_{\mathrm{ex}}\right)=T_{2}$, the following applies:

$$
\frac{U\left(\mathbf{R}_{1}\right)-U_{1}}{k_{B} T_{1}} \cong \frac{U\left(\mathbf{R}_{2}\right)-U_{2}}{k_{B} T_{2}} .
$$

Changing sign and taking the exponential this becomes Eq. (1), the condition that the two state points are isomorphic according to the original definition. ${ }^{27}$ Thus, as also stated in Sec. II, the original formulation of the isomorph theory is the first-order approximation to the new formulation.

\section{Strong virial potential-energy correlations for constant-density fluctuations}

The microscopic virial is defined ${ }^{10,38}$ by

$$
W(\mathbf{R}) \equiv-\frac{1}{3} \mathbf{R} \cdot \nabla U(\mathbf{R}) .
$$

Recall that at any state point the average of $W(\mathbf{R})$ (denoted by $W$ ) gives the contribution to the pressure from the interactions via the general equation of state $p V=N k_{B} T+W \cdot \cdot^{10,38}$

Below, we first show that the potential energy determines the virial, which implies that these quantities are strongly correlated. Next we calculate the proportionality constant of the virial potential-energy equilibrium fluctuations. In regard to the first objective, suppose two configurations are given, $\mathbf{R}_{a}$ and $\mathbf{R}_{b}$, which have the same density and the same potential energy, $U\left(\mathbf{R}_{a}\right)=U\left(\mathbf{R}_{b}\right)$. We conclude from Eq. (8) that $U\left(\lambda \mathbf{R}_{a}\right)=U\left(\lambda \mathbf{R}_{b}\right)$. Taking the derivative of this with respect to $\lambda$ results in $\mathbf{R}_{a} \cdot \nabla U\left(\lambda \mathbf{R}_{a}\right)=\mathbf{R}_{b} \cdot \nabla U\left(\lambda \mathbf{R}_{b}\right)$, which for $\lambda=1$ implies $W\left(\mathbf{R}_{a}\right)=W\left(\mathbf{R}_{b}\right)$. Thus any two configurations with same density and potential energy have the same virial. This means that $W$ is a function of $U$ and density, which implies perfect correlations between potential energy and virial at constant density. No realistic systems obey Eq. (8) with mathematical rigor, so in practice the correlations will not be perfect, but strong.

The constant of proportionality between the equilibrium virial and potential-energy fluctuations at a given state point is denoted by $\gamma$ and referred to as the density-scaling exponent; ${ }^{8,13,27} \gamma$ is characterized by

$$
\Delta W(t) \cong \gamma \Delta U(t) .
$$

Reference 27 defined $\gamma$ at any given state point by

$$
\gamma\left(\rho, S_{\mathrm{ex}}\right) \equiv\left(\frac{\partial \ln T}{\partial \ln \rho}\right)_{S_{\mathrm{ex}}}
$$

and derived the general fluctuation expression

$$
\gamma\left(\rho, S_{\mathrm{ex}}\right)=\frac{\langle\Delta W \Delta U\rangle}{\left\langle(\Delta U)^{2}\right\rangle} .
$$

Here the angular brackets denote canonical $N V T$ averages. Whenever Eq. (18) is obeyed to a good approximation, i.e., for Roskilde-simple systems, Eq. (20) implies that $\gamma$ of Eq. (19) is the same as that appearing in Eq. (18), ensuring consistency. We proceed to derive Eq. (18) from the definition of the density-scaling exponent Eq. (19) for Roskilde-simple systems.

As shown in the Appendix $W(\mathbf{R})=(\partial U(\mathbf{R}) / \partial \ln \rho)_{\tilde{\mathbf{R}}},{ }^{27}$ an expression which basically expresses that the microscopic virial is given by the work done to uniformly expand a given configuration. Substituting Eq. (14) into this expression leads to

$$
W(\mathbf{R})=\left(\frac{\partial U\left(\rho, S_{\mathrm{ex}}(\tilde{\mathbf{R}})\right)}{\partial \ln \rho}\right)_{\tilde{\mathbf{R}}} .
$$

Expanding to first order around the equilibrium values of virial and excess entropy at the state point in question leads to

$$
W(\mathbf{R}) \cong W+\left(\frac{\partial^{2} U}{\partial S_{\mathrm{ex}} \partial \ln \rho}\right)\left(S_{\mathrm{ex}}(\tilde{\mathbf{R}})-S_{\mathrm{ex}}\right) .
$$

Interchanging the order of the differentiations we get

$$
W(\mathbf{R})-W \cong\left(\frac{\partial T}{\partial \ln \rho}\right)_{S_{\mathrm{ex}}}\left(S_{\mathrm{ex}}(\tilde{\mathbf{R}})-S_{\mathrm{ex}}\right) .
$$

Eliminating $S_{\mathrm{ex}}(\tilde{\mathbf{R}})-S_{\text {ex }}$ from Eqs. (15) and (23) leads to

$$
W(\mathbf{R})-W \cong\left(\frac{\partial \ln T}{\partial \ln \rho}\right)_{S_{\mathrm{ex}}}(U(\tilde{\mathbf{R}})-U) .
$$

This can be rewritten as

$$
W(\mathbf{R}) \cong \gamma\left(\rho, S_{\mathrm{ex}}\right) U(\mathbf{R})+C\left(\rho, S_{\mathrm{ex}}\right)
$$

in which $\gamma\left(\rho, S_{\mathrm{ex}}\right)$ is the density-scaling exponent defined in Eq. (19) and $C\left(\rho, S_{\text {ex }}\right)=W-\gamma\left(\rho, S_{\text {ex }}\right) U$. For the constantdensity equilibrium fluctuations at a given state point Eq. (25) implies Eq. (18), i.e., strong virial potential-energy correlations.

\section{Single-parameter family of reduced-coordinate constant-potential-energy hypersurfaces}

A molecular dynamics may be formulated that is defined by geodesic motion on the constant-potential-energy hypersurface, so-called $N V U$ dynamics. ${ }^{39,40}$ For most quantities $N V U$ dynamics gives results that in the thermodynamic limit are identical to those of conventional Newtonian $N V T$ or $N V E$ dynamics. ${ }^{40}$ In reduced coordinates, the constant-potential-energy hypersurfaces are the sets defined by $\left\{\tilde{\mathbf{R}}^{\prime} \mid U\left(\rho^{-1 / 3} \tilde{\mathbf{R}}^{\prime}\right)=\right.$ Const. $\}$. These sets are generally parameterized by the two parameters specifying a thermodynamic state point, e.g., density and average potential energy, which in regard to $N V U$ dynamics results in the a priori expected situation of different dynamics at different state points. For a Roskilde-simple system, however, Eq. (14) implies that these sets are identical to the reduced-coordintate constantexcess-entropy hypersurfaces, i.e., parameterized by a single number, the excess entropy. ${ }^{27}$ This is consistent with the isomorph invariance of the dynamics.

\section{CONNECTING TO THE APPROACH OF SEC. II}

To establish the equivalence of the formulations of the new isomorph theory given in Secs. II and III, respectively, we consider two state points $\left(\rho_{1}, T_{1}\right)$ and $\left(\rho_{2}, T_{2}\right)$ with the same excess entropy, $S_{\text {ex }}\left(\rho_{1}, T_{1}\right)=S_{\text {ex }}\left(\rho_{2}, T_{2}\right)$. If $\mathbf{R}_{1}$ is a typical configuration of the first state point and similarly for $\mathbf{R}_{2}$, by definition of the microscopic entropy function (Eqs. (9) 
and (10)) recalling that $U\left(\rho, S_{\mathrm{ex}}\right)$ and $S_{\mathrm{ex}}(\rho, U)$ are the thermodynamic functions relating state point averages we have

$$
\begin{aligned}
& U\left(\mathbf{R}_{1}\right)=U\left(\rho_{1}, S_{\mathrm{ex}}\left(\rho_{1}, U\left(\mathbf{R}_{1}\right)\right)\right), \\
& U\left(\mathbf{R}_{2}\right)=U\left(\rho_{2}, S_{\mathrm{ex}}\left(\rho_{2}, U\left(\mathbf{R}_{2}\right)\right)\right) .
\end{aligned}
$$

Writing as in Sec. II for brevity $U\left(\mathbf{R}_{1}\right)=U_{1}$, etc., if the two configurations have the same reduced coordinates, since Eq. (13) implies $S_{\text {ex }}\left(\rho_{1}, U_{1}\right)=S_{\text {ex }}\left(\rho_{2}, U_{2}\right)$, we have that

$$
U_{2}=U\left(\rho_{2}, S_{\mathrm{ex}}\left(\rho_{2}, U_{2}\right)\right)=U\left(\rho_{2}, S_{\mathrm{ex}}\left(\rho_{1}, U_{1}\right)\right) .
$$

Comparing to Eq. (4) leads to the identification

$$
f_{1}\left(\rho_{2}, U_{1}\right)=U\left(\rho_{2}, S_{\mathrm{ex}}\left(\rho_{1}, U_{1}\right)\right) .
$$

To validate this expression we calculate the ratio $T_{2} / T_{1}$, which according to Sec. II should be given by $T_{2} / T_{1}$ $=\left(\partial f_{1} / \partial U_{1}\right)_{\rho_{1}, \rho_{2}}$. Since the two state points have same excess entropy, below denoted by $S_{\text {ex }}$, and since $\rho_{1}$ by virtue of its role as reference density is constant throughout, Eq. (28) implies

$$
\left(\frac{\partial f_{1}}{\partial U_{1}}\right)_{\rho_{1}, \rho_{2}}=\left(\frac{\partial U\left(\rho_{2}, S_{\mathrm{ex}}\right)}{\partial S_{\mathrm{ex}}}\right)_{\rho_{2}}\left(\frac{\partial S_{\mathrm{ex}}}{\partial U_{1}}\right)_{\rho_{1}} .
$$

From the thermodynamic definition of temperature, this gives the required

$$
\left(\frac{\partial f_{1}}{\partial U_{1}}\right)_{\rho_{2}}=\frac{T_{2}}{T_{1}}
$$

\section{CONCLUDING REMARKS}

Appendix A of the original isomorph paper Ref. 27 showed that points B, C, and D of Sec. III are equivalent, i.e., if any one of these three quite different characterizations of a given system applies, the two others follow by necessity. In that paper, isomorphs were defined from the condition Eq. (1), and the reduced-unit isomorph invariance of structure and dynamics was derived from this equation. Likewise, Eq. (1) was shown to imply that isomorphs are configurational adiabats. In contrast, we have here defined the isomorphs as the configurational adiabats and showed that structure and dynamics are invariant along these.

Section III D discussed the constant-potential-energy hypersurface characterization of Roskilde-simple systems: In reduced-coordinate space these hypersurfaces constitute a single-parameter family parameterized by the value of the microscopic excess entropy function. In Ref. 27 this property was discussed in a slightly different language, noting that these high-dimensional hypersurfaces are identical along the isomorphs (a property that via $N V U$ dynamics immediately implies isomorph invariance of the dynamics). We here wish to point out that this characterization is closely related to the present paper's definition of Roskilde simple systems. Suppose that $\mathbf{R}_{a}$ and $\mathbf{R}_{b}$ are two configurations of same density, which have the same potential energy, $U\left(\mathbf{R}_{a}\right)=U\left(\mathbf{R}_{b}\right)$. Then these two configurations belong to the same reduced-coordinate constant-potential-energy hypersurface $\tilde{\Omega}$. Because both of the uniformly scaled configu- rations, $\lambda \mathbf{R}_{a}$ and $\lambda \mathbf{R}_{b}$, have the same reduced coordinates as the original configuration, these scaled ones are located on the same reduced-coordinate constant-potential-energy hypersurface $\tilde{\Omega}$. This implies that they have the same potential energy, i.e., $U\left(\lambda \mathbf{R}_{a}\right)=U\left(\lambda \mathbf{R}_{b}\right)$. We conclude that Eq. (8) applies whenever the reduced-coordinate constant-potential-energy hypersurfaces constitute a single-parameter family throughout the thermodynamic phase diagram.

In the present treatment Eq. (16) and thus Eq. (1) is derived by a first-order expansion of the fundamental equation, Eq. (14), which implies invariance of structure and dynamics when isomorphs are defined as the configurational adiabats. The hidden-scale-invariance identity Eq. (2) is replaced by Eq. (14), and the roles of the two abstract functions $h(\rho)$ and $\tilde{\Phi}(\tilde{\mathbf{R}})$ in Eq. (2) are taken over by the temperature $T(\rho$, $S_{\text {ex }}$ ) and the microscopic excess entropy function $S_{\text {ex }}(\tilde{\mathbf{R}})$. In practice, the main changes compared to the original isomorph theory are that $C_{V}$ is only isomorph invariant to first order ${ }^{31}$ because the proof of its isomorph invariance was based on Eq. (16) ${ }^{27}$ and that, likewise, the density-scaling exponent is only to first order a function merely of the density. An implication is that the density-scaling phenomenon involves a hierarchy of approximations: In the simplest case, the density scaling exponent $\gamma$ is constant, implying that the isomorphs (=isochrones) are given by $\rho^{\gamma} / T=$ Const. In the more general case described by the hidden scale invariance identity of the original formulation of the isomorph theory based on Eq. (2), the isomorphs are given by $h(\rho) / T=$ Const. corresponding to a density-scaling exponent (Eq. (19)) that may vary throughout the phase diagram, but only as a function of density. Finally, the present formulation allows for the density-scaling exponent to vary more generally.

For a pair-potential system with $v(r)=\sum_{n} \varepsilon_{n}(r / \sigma)^{-n}$, because of the structural invariance along an isomorph in reduced coordinates, one has $U\left(\rho, S_{\mathrm{ex}}\right)=\sum_{n} C_{n}\left(S_{\mathrm{ex}}\right) \rho^{n / 3} \cdot 33,34$ This equation of state, which was previously derived by Rosenfeld assuming quasiuniversality, ${ }^{41}$ implies $T\left(\rho, S_{\mathrm{ex}}\right)=$ $\sum_{n} C_{n}^{\prime}\left(S_{\mathrm{ex}}\right) \rho^{n / 3}$. For the LJ system, this leads to the isomorph equation $\left[\alpha_{12}\left(S_{\mathrm{ex}}\right) \rho^{4}-\alpha_{6}\left(S_{\mathrm{ex}}\right) \rho^{2}\right] / T=$ Const., which is validated numerically in Fig. 3.

In summary, this paper proposes a new definition of Roskilde-simple systems, Eq. (7). Equivalently, one may use Eq. (8) as the new definition. The new isomorph theory is simpler than the original one and its predictions are more accurate. The original isomorph theory is recovered as a firstorder approximation to the new one. The new definition does not change the class of Roskilde-simple systems. This class is still believed to include most van der Waals bonded and metallic solids and liquids, as well as the weakly ionic or dipolar systems, and exclude most hydrogen-bonded and covalently bonded systems, as well as the strongly ionic or dipolar systems.

\section{ACKNOWLEDGMENTS}

The center for viscous liquid dynamics "Glass and Time" is sponsored by the Danish National Research Foundation via Grant No. DNRF61. 


\section{APPENDIX: EXCESS THERMODYNAMICS AND THE CONFIGURATION-SPACE MICROCANONICAL EXPRESSION FOR THE EXCESS ENTROPY}

Consider a system of $N$ identical particles in volume $V$ with density $\rho=N / V$. The particle coordinates are given by the $3 N$-dimensional vector $\mathbf{R} \equiv\left(\mathbf{r}_{1}, \ldots, \mathbf{r}_{N}\right)$ and the corresponding reduced (dimensionless) coordinate vector is defined by $\tilde{\mathbf{R}} \equiv \rho^{1 / 3} \mathbf{R}$. This Appendix derives an expression for the microscopic virial, summarizes the definition of excess (configurational) thermodynamic quantities, and derives the microcanonical expression for the excess entropy at the state point with density $\rho$ and average potential energy $U$,

$$
S_{\mathrm{ex}}(\rho, U) / k_{B}=-N \ln N+\ln \left(\operatorname{Vol}\left\{\tilde{\mathbf{R}} \mid U\left(\rho^{-1 / 3} \tilde{\mathbf{R}}\right)<U\right\}\right) .
$$

Here "Vol" refers to the volume of the set in question, which is the $\tilde{\mathbf{R}}$ integral of the unity function over all configurations $\mathbf{R}=\rho^{-1 / 3} \tilde{\mathbf{R}}$ with potential energy below $U$, i.e., obeying $U(\mathbf{R})<U$.

\section{An expression for the microscopic virial}

Consider the infinitesimal uniform expansion $\mathbf{R} \rightarrow(1+d \lambda) \mathbf{R}$. The relative volume change is $d V / V$ $=(1+d \lambda)^{3}-1=3 d \lambda$, which implies $d \ln \rho=d \rho / \rho$ $=-d V / V=-3 d \lambda$, i.e., $d \lambda=-(1 / 3) d \ln \rho$. The change of the configuration vector is given by $d \mathbf{R}=d \lambda \mathbf{R}$, so the change of the potential energy is $d U(\mathbf{R})=d \lambda \mathbf{R} \cdot \nabla U(\mathbf{R})$ $=-(1 / 3) d \ln \rho \mathbf{R} \cdot \nabla U(\mathbf{R})$. Comparing to the definition of the virial Eq. (17) we get $d U(\mathbf{R})=d \ln \rho W(\mathbf{R})$. The reduced coordinate $\tilde{\mathbf{R}}$ is constant during the uniform expansion, so we conclude that

$$
W(\mathbf{R})=\left(\frac{\partial U(\mathbf{R})}{\partial \ln \rho}\right)_{\tilde{\mathbf{R}}} .
$$

\section{Excess thermodynamic quantities}

Recall from statistical mechanics that if the momentum degrees of freedom are denoted by $\mathbf{P} \equiv\left(\mathbf{p}_{1}, \ldots, \mathbf{p}_{N}\right)$ and $H(\mathbf{P}, \mathbf{R})$ is the Hamiltonian, the Helmholtz free energy $F$ is given by the classical partition function as follows (where $\left.\beta \equiv 1 / k_{B} T\right):{ }^{10}$

$$
e^{-\beta F}=\frac{1}{N !} \int \frac{d \mathbf{P} d \mathbf{R}}{h^{3 N}} e^{-\beta H(\mathbf{P}, \mathbf{R})} .
$$

The appearances of Planck's constant $h$ and the indistinguishability factor $1 / N$ ! ensure proper correspondence to quantum mechanics. These factors are conveniently absorbed by writing $F=F_{\text {id }}+F_{\text {ex }}$ in which $F_{\text {id }}$ is the free energy of an ideal gas at the same density and temperature, $F_{\text {id }}=$ $N k_{B} T\left(\ln \left(\Lambda^{3} \rho\right)-1\right)$ where $\Lambda=h / \sqrt{2 \pi m k_{B} T}$ is the thermal de Broglie wavelength ( $m$ is the particle mass). ${ }^{10}$ The result of these manipulations is that the excess free energy $F_{\text {ex }}$ is given by

$$
e^{-\beta F_{\mathrm{ex}}}=\int \frac{d \mathbf{R}}{V^{N}} e^{-\beta U(\mathbf{R})} .
$$

In the case of free particles, $U=0$, we get $F_{\text {ex }}=0$ as required for consistency. Note that there is no $1 / N$ ! factor in Eq. (A4), so $F_{\text {ex }}$ is formally the free energy of a system of distinguishable particles with no momentum coordinates.

Due to the separation $F=F_{\text {id }}+F_{\text {ex }}$, all thermodynamic quantities that are derivatives of $F$ separate into an ideal-gas contribution and an "excess" contribution. For instance, for the entropy one has $S=S_{\text {id }}+S_{\text {ex }}$ in which $S_{\text {ex }}=-\left(\partial F_{\text {ex }} / \partial T\right)_{\rho}$, the isochoric specific heat separates into a sum of two terms and the well-known relation $C_{V}^{\mathrm{ex}}=\left(\partial S_{\mathrm{ex}} / \partial \ln T\right)_{\rho}$ applies, etc.

The excess entropy obeys $S_{\text {ex }}<0$ because a liquid is always more ordered than an ideal gas at the same density and temperature. As temperature goes to infinity, the system approaches the complete chaos of an ideal gas, so $S_{\mathrm{ex}} \rightarrow 0$ for $T \rightarrow \infty$ at fixed density. The relation between excess entropy, potential energy, and temperature is the usual one, i.e.,

$$
\left(\frac{\partial S_{\mathrm{ex}}}{\partial U}\right)_{\rho}=\frac{1}{T}
$$

For the pressure the equation characterizing the average virial $W$, i.e., the average of $W(\mathbf{R})$ of Eq. (17), is $p V=N k_{B} T$ $+W .{ }^{10}$ This implies that $p=p_{\text {id }}+W / V$. Thus the excess pressure is $W / V$, a quantity that in terms of $F_{\mathrm{ex}}$ is given by $W / V=-\left(\partial F_{\text {ex }} / \partial V\right)_{T}$.

\section{The microcanonical expression for the excess entropy}

The Heaviside theta function is denoted by $\Theta(x)$; recall that this function is unity for positive arguments and zero for negative. The dimensionless volume of the set of configurations with potential energy less than $U$ is denoted by $\Omega(U)$ and given by

$$
\Omega(U)=\int \frac{d \mathbf{R}}{V^{N}} \Theta(U-U(\mathbf{R})) .
$$

If $X_{i}$ is one of the $3 N$ particle coordinates and $\partial_{j} \equiv \partial / \partial X_{j}$, the microcanonical average of $X_{i} \partial_{j} U(\mathbf{R})$ is by definition

$$
\left\langle X_{i} \partial_{j} U(\mathbf{R})\right\rangle_{\mathrm{mc}}=\frac{\int\left(d \mathbf{R} / V^{N}\right) X_{i}\left(\partial_{j} U(\mathbf{R})\right) \delta(U-U(\mathbf{R}))}{\int\left(d \mathbf{R} / V^{N}\right) \delta(U-U(\mathbf{R}))} .
$$

Following Pauli, ${ }^{42}$ via the fact that $\Theta^{\prime}(x)=\delta(x)$ and a partial integration we get for the numerator

$$
\begin{aligned}
\int & \frac{d \mathbf{R}}{V^{N}} X_{i}\left(\partial_{j} U(\mathbf{R})\right) \delta(U-U(\mathbf{R})) \\
= & \frac{d}{d U} \int \frac{d \mathbf{R}}{V^{N}} X_{i}\left(\partial_{j} U(\mathbf{R})\right) \Theta(U-U(\mathbf{R})) \\
= & \frac{d}{d U} \int \frac{d \mathbf{R}}{V^{N}} X_{i}\left(\partial_{j}(U(\mathbf{R})-U)\right) \Theta(U-U(\mathbf{R})) \\
= & -\frac{d}{d U} \delta_{i j} \int \frac{d \mathbf{R}}{V^{N}}(U(\mathbf{R})-U) \Theta(U-U(\mathbf{R})) \\
= & \delta_{i j} \int \frac{d \mathbf{R}}{V^{N}} \Theta(U-U(\mathbf{R})) \\
= & \delta_{i j} \Omega(U) .
\end{aligned}
$$


The denominator of Eq. (A7) is $\Omega^{\prime}(U)$, so all together we get

$$
\left\langle X_{i} \partial_{j} U(\mathbf{R})\right\rangle_{\mathrm{mc}}=\delta_{i j} \frac{\Omega(U)}{\Omega^{\prime}(U)} .
$$

Next, the canonical average of $X_{i} \partial_{j} U(\mathbf{R})$ is calculated. If $Z=\int d \mathbf{R} / V^{N} \exp (-\beta U(\mathbf{R}))$ is the partition function we have $\left\langle X_{i} \partial_{j} U(\mathbf{R})\right\rangle_{\text {can }}=\int d \mathbf{R} / V^{N} X_{i}\left(\partial_{j} U(\mathbf{R})\right) \exp (-\beta U(\mathbf{R})) /$ $Z=-k_{B} T \int d \mathbf{R} / V^{N} X_{i} \partial_{j} \exp (-\beta U(\mathbf{R})) / Z$, which via a partial integration gives $k_{B} T \delta_{i j}$. Since averages are ensemble independent (in contrast to fluctuations), Eq. (A9) implies

$$
\frac{\Omega(U)}{\Omega^{\prime}(U)}=k_{B} T .
$$

Combined with Eq. (A5) this implies that $\left(\partial S_{\mathrm{ex}} / \partial U\right)_{\rho}=1 / T$ $=k_{B} d \ln \Omega(U) / d U$, i.e.,

$$
S_{\text {ex }}=k_{B} \ln \Omega(U)+\text { Const. }
$$

The constant, which is a function of density, is determined from the above-mentioned boundary condition $S_{\text {ex }} \rightarrow 0$ for $T \rightarrow \infty$ at constant density. From Eq. (A6) we conclude that the constant is zero. Rewriting finally the definition of $\Omega(U)$ as an integral over the reduced coordinate vector $\tilde{\mathbf{R}}$ leads to Eq. (A1).

${ }^{1}$ S. A. Rice and P. Gray, The Statistical Mechanics of Simple Liquids (Interscience, New York, 1965).

${ }^{2}$ H. N. V. Temperley, J. S. Rowlinson, and G. S. Rushbrooke, Physics of Simple Liquids (Wiley, New York, 1968).

${ }^{3}$ J. S. Rowlinson and B. Widom, Molecular Theory of Capillarity (Clarendon, Oxford, 1982).

${ }^{4}$ D. Chandler, Introduction to Modern Statistical Mechanics (Oxford University Press, 1987).

${ }^{5}$ J.-L. Barrat and J.-P. Hansen, Basic Concepts for Simple and Complex Liquids (Cambridge University Press, 2003).

${ }^{6}$ P. G. Debenedetti, AICHE J. 51, 2391 (2005).

${ }^{7}$ N. P. Bailey, U. R. Pedersen, N. Gnan, T. B. Schrøder, and J. C. Dyre, J. Chem. Phys. 129, 184507 (2008).

${ }^{8}$ N. P. Bailey, U. R. Pedersen, N. Gnan, T. B. Schrøder, and J. C. Dyre, J. Chem. Phys. 129, 184508 (2008).

${ }^{9}$ B. Bagchi and C. Chakravarty, J. Chem. Sci. 122, 459 (2010).

${ }^{10}$ J.-P. Hansen and I. R. McDonald, Theory of Simple Liquids: With Applications to Soft Matter, 4th ed. (Academic, New York, 2013).

${ }^{11}$ M. J. Pond, J. R. Errington, and T. M. Truskett, J. Chem. Phys. 134, 081101 (2011).

${ }^{12}$ S. Prasad and C. Chakravarty, J. Chem. Phys. 140, 164501 (2014).

${ }^{13}$ J. C. Dyre, J. Phys. Chem. B 118, 10007 (2014).

${ }^{14}$ E. H. Abramson, J. Phys. Chem. B 118, 11792 (2014).
${ }^{15}$ A. Malins, J. Eggers, and C. P. Royall, J. Chem. Phys. 139, 234505 (2013).

${ }^{16}$ E. Flenner, H. Staley, and G. Szamel, Phys. Rev. Lett. 112, 097801 (2014).

${ }^{17}$ A. Henao, S. Pothoczki, M. Canales, E. Guardia, and L. Pardo, J. Mol. Liquids 190, 121 (2014).

${ }^{18}$ S. Pieprzyk, D. M. Heyes, and A. C. Branka, Phys. Rev. E 90, 012106 (2014).

${ }^{19} \mathrm{U}$. Buchenau, "Thermodynamics and dynamics of the inherent states at the glass transition," J. Non-Cryst. Solids (published online).

${ }^{20}$ J. Fernandez and E. R. Lopez, in Experimental Thermodynamics: Advances in Transport Properties of Fluids (Royal Society of Chemistry, 2014), Chap. 9.3, pp. 307-317.

${ }^{21}$ J. W. P. Schmelzer and T. V. Tropin, "Kinetic criteria of glassformation, pressure dependence of the glass-transition temperature, and the Prigogine-Defay ratio," J. Non-Cryst. Solids (published online).

${ }^{22}$ C. M. Roland, S. Hensel-Bielowka, M. Paluch, and R. Casalini, Rep. Prog. Phys. 68, 1405 (2005)

${ }^{23}$ G. Floudas, M. Paluch, A. Grzybowski, and K. Ngai, Molecular Dynamics of Glass-Forming Systems: Effects of Pressure (Springer, Berlin, 2011).

${ }^{24}$ K. L. Ngai, R. Casalini, S. Capaccioli, M. Paluch, and C. M. Roland, J. Phys. Chem. B 109, 17356 (2005).

${ }^{25}$ L. A. Roed, D. Gundermann, J. C. Dyre, and K. Niss, J. Chem. Phys. 139, 101101 (2013).

${ }^{26}$ A. R. Ubbelohde, Melting and Crystal Structure (Clarendon, Oxford, 1965).

${ }^{27}$ N. Gnan, T. B. Schrøder, U. R. Pedersen, N. P. Bailey, and J. C. Dyre, J. Chem. Phys. 131, 234504 (2009).

${ }^{28}$ J. C. Dyre, Phys. Rev. E 87, 022106 (2013).

${ }^{29}$ U. R. Pedersen, J. Chem. Phys. 139, 104102 (2013).

${ }^{30}$ J. C. Dyre, Phys. Rev. E 88, 042139 (2013).

${ }^{31}$ N. P. Bailey, L. Bøhling, A. A. Veldhorst, T. B. Schrøder, and J. C. Dyre, J. Chem. Phys. 139, 184506 (2013).

${ }^{32}$ All simulations were performed using a molecular dynamics code optimized for NVIDIA graphics cards, which is available as open source code at http://rumd.org.

${ }^{33}$ L. Bøhling, T. S. Ingebrigtsen, A. Grzybowski, M. Paluch, J. C. Dyre, and T. B. Schrøder, New J. Phys. 14, 113035 (2012).

${ }^{34}$ T. S. Ingebrigtsen, L. Bøhling, T. B. Schrøder, and J. C. Dyre, J. Chem. Phys. 136, 061102 (2012).

${ }^{35}$ A. Barros de Oliveira, P. A. Netz, T. Colla, and M. C. Barbosa, J. Chem. Phys. 124, 084505 (2006).

${ }^{36}$ L. D. Landau and E. M. Lifshitz, Statistical Physics (Pergamon, Oxford, 1958).

${ }^{37} \mathrm{C}$. Rovelli, "Why do we remember the past and not the future? The "time oriented coarse graining' hypothesis," preprint arXiv:1407.3384 (2014).

${ }^{38}$ M. P. Allen and D. J. Tildesley, Computer Simulation of Liquids (Oxford Science Publications, 1987).

${ }^{39}$ T. S. Ingebrigtsen, S. Toxvaerd, O. J. Heilmann, T. B. Schrøder, and J. C. Dyre, J. Chem. Phys. 135, 104101 (2011).

${ }^{40}$ T. S. Ingebrigtsen, S. Toxvaerd, T. B. Schrøder, and J. C. Dyre, J. Chem. Phys. 135, 104102 (2011).

${ }^{41}$ Y. Rosenfeld, Phys. Rev. A 26, 3633 (1982).

${ }^{42}$ W. Pauli, Pauli Lectures on Physics. Volume 4. Statistical Mechanics (MIT Press, Cambridge, MA, 1973). 